\title{
Decursin and Doxorubicin Are in Synergy for the Induction of Apoptosis via STAT3 and/or mTOR Pathways in Human Multiple Myeloma Cells
}

\author{
Jinsil Jang, ${ }^{1}$ Soo-Jin Jeong, ${ }^{1,2}$ Hee-Young Kwon, ${ }^{1}$ Ji Hoon Jung, ${ }^{1}$ Eun Jung Sohn, ${ }^{1}$ \\ Hyo-Jung Lee, ${ }^{1,3}$ Ji-Hyun Kim, ${ }^{1}$ Sun-Hee Kim, ${ }^{1}$ Jin Hyoung Kim, ${ }^{1}$ and Sung-Hoon Kim ${ }^{1}$ \\ ${ }^{1}$ Cancer Preventive Material Development Research Center, College of Oriental Medicine, Kyung Hee University, \\ 1 Hoegi-dong, Dongdaemun-gu, Seoul 131-701, Republic of Korea \\ ${ }^{2}$ Basic Herbal Research Group, Korea Institute of Oriental Medicine, Daejeon 305-811, Republic of Korea \\ ${ }^{3}$ Medical Genomics Research Center, Korea Research Institute of Bioscience and Biotechnology, Daejeon 305-806, Republic of Korea \\ Correspondence should be addressed to Sung-Hoon Kim; sungkim7@khu.ac.kr
}

Received 5 September 2012; Revised 16 March 2013; Accepted 1 April 2013

Academic Editor: Cheppail Ramachandran

Copyright (C) 2013 Jinsil Jang et al. This is an open access article distributed under the Creative Commons Attribution License, which permits unrestricted use, distribution, and reproduction in any medium, provided the original work is properly cited.

\begin{abstract}
Background. Combination cancer therapy is one of the attractive approaches to overcome drug resistance of cancer cells. In the present study, we investigated the synergistic effect of decursin from Angelica gigas and doxorubicin on the induction of apoptosis in three human multiple myeloma cells. Methodology/Principal Findings. Combined treatment of decursin and doxorubicin significantly exerted significant cytotoxicity compared to doxorubicin or decursin in U266, RPMI8226, and MM.1S cells. Furthermore, the combination treatment enhanced the activation of caspase- 9 and -3 , the cleavage of PARP, and the sub G1 population compared to either drug alone in three multiple myeloma cells. In addition, the combined treatment downregulated the phosphorylation of mTOR and its downstream S6K1 and activated the phosphorylation of ERK in three multiple myeloma cells. Furthermore, the combined treatment reduced mitochondrial membrane potential, suppressed the phosphorylation of JAK2, STAT3, and Src, activated SHP-2, and attenuated the expression of cyclind-D1 and survivin in U266 cells. Conversely, tyrosine phosphatase inhibitor pervanadate reversed STAT3 inactivation and also PARP cleavage and caspase-3 activation induced by combined treatment of doxorubicin and decursin in U266 cells. Conclusions/Significance. Overall, the combination treatment of decursin and doxorubicin can enhance apoptotic activity via mTOR and/or STAT3 signaling pathway in multiple myeloma cells.
\end{abstract}

\section{Introduction}

Multiple myeloma is a neoplasm of terminally differentiated neoplastic B cells (plasma cells), accounting for about 10\% of all hematologic malignancies [1-3]. Multiple myeloma is characterized by the infiltration and accumulation of monocytic plasma cells in the bone marrow [4]. Although various chemotherapeutic agents such as thalidomide [5], bortezomib [6], and lenalidomide [7] are successful in initial chemotherapy of multiple myeloma, the patients ultimately become drug resistance [4].

Recently, many groups reported that combination therapy is an effective process to overcome drug resistance of various types of human malignant diseases, including multiple myeloma. For instance, Stein and colleagues reported that milatuzumab, a humanized anti-CD74 monoclonal antibody, improved the response of multiple myeloma to treatment with bortezomib, doxorubicin, or dexamethasone [8]. Chauhan and colleagues demonstrated that 2-methoxyestradiol (2-ME), an estrogen derivative, enhanced dexamethasone-induced apoptosis [9]. In addition, Sanchez and colleagues showed that the proteasome inhibitor CEP-18770 was able to augment the antimyeloma activity of bortezomib and melphalan [10].

Angelica gigas Nakai (Umbelliferae) has been used in traditional oriental medicine for the prevention and treatment of blood diseases including anemia as a tonic agent [11]. Its major compound decursin exerted antitumor activity by apoptosis induction or angiogenesis inhibition in various 
cancer cells including prostate, bladder, and colon cancer, and leukemia [12-14]. Also, our group reported that decursin has a protective effect on neurotoxicity and nephrotoxicity in normal cells via activation of antioxidative enzymes $[15,16]$ and also decursin-induced apoptosis through inhibition of STAT3 signaling pathway in multiple myeloma U266 cells [17].

Also, the mammalian target of rapamycin (mTOR), a serine/threonine (ser/thr) protein kinase, regulates cell growth mediated by interaction of signals from growth factors, and its downstream protein ribosomal S6 kinas (S6 K1) also plays a crucial role in cell cycle progression. Thus, inhibition of mTOR pathway to induce apoptosis is an attractive target for cancer therapy [18-20].

Thus, in the present study, we investigated the synergistic effects of decursin and doxorubicin combination (Figure 1) for multiple myeloma treatment. The concurrent treatment of decursin and doxorubicin switched on mitochondriagoverned, apoptotic machinery in multiple myeloma cells. In addition, we suggest that combination of decursin- and doxorubicin-induced apoptosis through the suppression of the mTOR/S6K1 and, or STAT3 signaling pathway.

\section{Materials and Methods}

2.1. Reagents. Decursin was extracted and purified as described previously $[21,22]$. The purity was determined to be $\sim 98.6 \%$. Doxorubicin hydrochloride was purchased from Sigma (St. Louis, MO, USA). Both decursin and doxorubicin were dissolved in dimethyl sulfoxide (DMSO). In all experiment, DMSO concentration was kept below $0.2 \%(\mathrm{v} / \mathrm{v})$ to remove the effect of vehicle DMSO.

2.2. Cell Culture. U266 [23], MM.1S [24], and RPMI8226 cells were obtained from American Type Culture Collection and maintained in RPMI 1640 supplemented with 10\% fetal bovine serum (FBS, Welgene, Korea), nonessential amino acids, pyruvate, glutamine, vitamins, penicillin $(100 \mathrm{U} / \mathrm{mL})$, and streptomycin $(100 \mathrm{~g} / \mathrm{mL})$. The cells were routinely tested for mycoplasma contamination to ensure that only contaminated negative cells were used.

2.3. Cytotoxicity Assay. The cytotoxicity of decursin and/or doxorubicin was measured by 2,3-bis-(2-methoxy-4-nitro-5sulfophenyl)- $2 \mathrm{H}$-tetrazolium-5-carboxanilide (XTT) colorimetric assay (Sigma Chemical Co., St. Louis, Mo, USA). U266, RPMI8226 or MM1.S cells were seeded onto 96-well microplates at a density of $2 \times 10^{4}$ cells per well in $100 \mu \mathrm{L}$ of growth medium with various concentrations of decursin and/or doxorubicin. XTT working solution was prepared just prior to culture application by mixing $1 \mathrm{~mL}$ of XTT stock solution $(1 \mathrm{mg} / \mathrm{mL}$ in $\mathrm{PBS})$ with $10 \mu \mathrm{L}$ of phenazine methosulfate (PMS) $\left(1.53 \mathrm{mg} / \mathrm{mL}\right.$ in PBS). After incubation at $37^{\circ} \mathrm{C}$ in a humidified incubator for $24 \mathrm{~h}$, a $50 \mu \mathrm{L}$ of XTT working solution was added to each well. Cells were incubated at $37^{\circ} \mathrm{C}$ for $2 \mathrm{~h}$, and the optical density was measured using microplate reader (Sunrise, TECAN) at $450 \mathrm{~nm}$. Cell viability was calculated as a percentage of viable cells in drug-treated group versus untreated control by a following equation. Cell viability $(\%)=[\mathrm{OD}(\mathrm{drug})-\mathrm{OD}(\mathrm{Blank})] /[\mathrm{OD}$ (Control) - OD $($ Blank) $] \times 100$. Wells containing XTT reagent in the absence of cells were included to verify that the reagent did not interfere with the test.

2.4. Combination Index (CI) Calculation. Cells were treated with decursin $(40 \mu \mathrm{M}$ or $80 \mu \mathrm{M})$ and doxorubicin $(0.5$ or $1 \mu \mathrm{M})$. The CI was determined by the Chou-Talalay method and CalcuSyn software (Biosoft, Ferguson, MO, USA). A CI of less than 1 was considered synergistic based on Zhao's principle [25].

2.5. Live/Dead Assay. To measure apoptosis, we used the live and dead assay (Molecular Probes), which determines intracellular esterase activity and plasma membrane integrity. In brief, $1 \times 10^{6}$ cells were incubated with decursin and/or doxorubicin for $24 \mathrm{~h}$. Cells were stained with the live and dead reagent ( $5 \mu \mathrm{M}$ ethidium homodimer and $5 \mu \mathrm{M}$ calcein-AM) and then incubated at $37^{\circ} \mathrm{C}$ for $30 \mathrm{~min}$. Cells were analyzed under Axio vision 4.0 fluorescence microscope (Carl Zeiss Inc., USA).

2.6. Cell Cycle Analysis. To determine apoptosis, cell cycle analysis was performed as previously described [26]. Cells $\left(1 \times 10^{6}\right)$ treated with decursin and/or doxorubicin were harvested, washed twice with cold PBS, and fixed in 75\% ethanol at $-20^{\circ} \mathrm{C}$. The fixed cells were resuspended in $100 \mu \mathrm{L}$ of PBS containing $10 \mu \mathrm{L}$ of RNase $\mathrm{A}(10 \mathrm{mg} / \mathrm{mL})$ and incubated for $1 \mathrm{~h}$ at $37^{\circ} \mathrm{C}$. The cells were stained by adding $400 \mu \mathrm{L}$ of propidium iodide $(50 \mu \mathrm{g} / \mathrm{mL})$ for $30 \mathrm{~min}$ at room temperature in the dark. The DNA contents of stained cells were analyzed using CellQuest Software with the FACSCalibur (Becton Dickinson, Heidelberg, Germany) flow cytometry.

2.7. TdT-Mediated dUTP Nick End Labelling (TUNEL) Assay. Flow cytometric analysis was carried out using an in situ cell death detection reagent (Roche Molecular Biochemicals, Mannheim, Germany) as described by the manufacturer. U266 cells $\left(1 \times 10^{6}\right)$ were treated with decursin and/or doxorubicin for $24 \mathrm{~h}$ at $37^{\circ} \mathrm{C}$. The cells were fixed in $4 \%$ paraformaldehyde in PBS at room temperature for $60 \mathrm{~min}$ then washed in PBS and permeability enhanced by treatment with $0.1 \%$ Triton X-100 in $0.1 \%$ sodium citrate for 2 min on ice. Cells were washed twice in PBS and resuspended in TUNEL reaction mixture with TUNEL enzyme and incubated for $60 \mathrm{~min}$ at $37^{\circ} \mathrm{C}$ in a humidified atmosphere in the dark. Cells were washed three times with PBS and analysed by flow cytometry (FACSCalibur, BD Biosciences).

2.8. Western Blotting. Cells $\left(1 \times 10^{6}\right)$ treated with decursin and/or doxorubicin were harvested and washed with cold PBS. Cell pellets were lysed in $30 \mu \mathrm{L}$ of lysis buffer $(50 \mathrm{mM}$ Tris- $\mathrm{HCl}, \mathrm{pH} 7.4,150 \mathrm{mM} \mathrm{NaCl}, 1 \%$ Triton X-100, $0.1 \%$ SDS, $1 \mathrm{mM}$ EDTA, $1 \mathrm{mM} \mathrm{Na} \mathrm{VO}_{4}, 1 \mathrm{mM} \mathrm{NaF}$, and protease inhibitor cocktail) for $30 \mathrm{~min}$ on ice. Lysates were centrifuged at $13,000 \times \mathrm{g}$ for $20 \mathrm{~min}$ at $4^{\circ} \mathrm{C}$, and the protein contents in the supernatants were measured by using a Bio-Rad detergent compatible (DC) protein assay kit II. Proteins $(20 \mu \mathrm{g} /$ well $)$ 


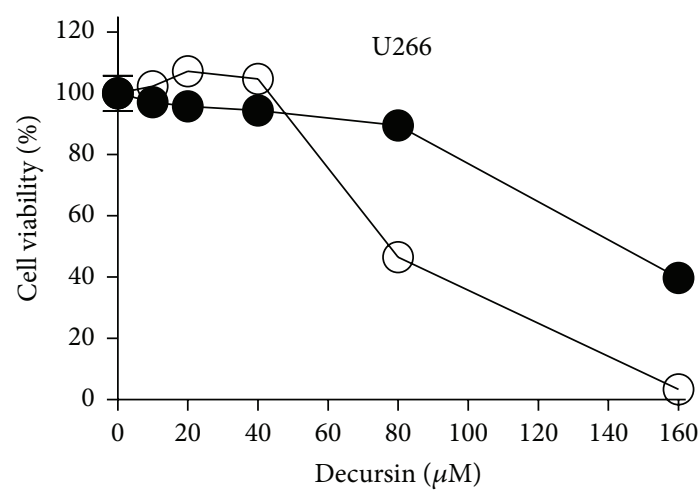

(a)

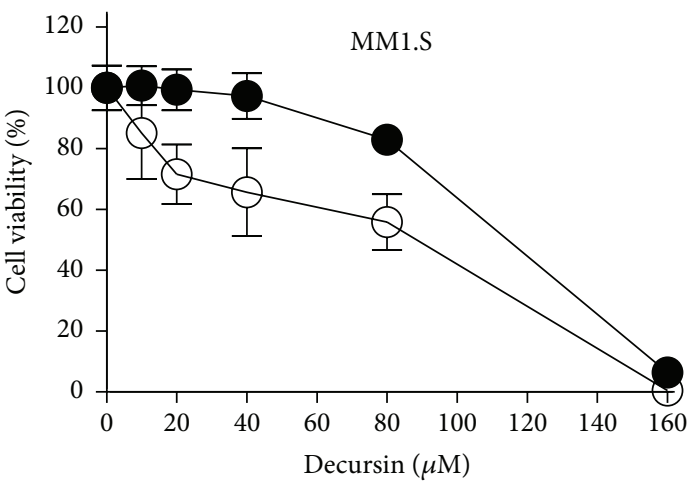

(c)

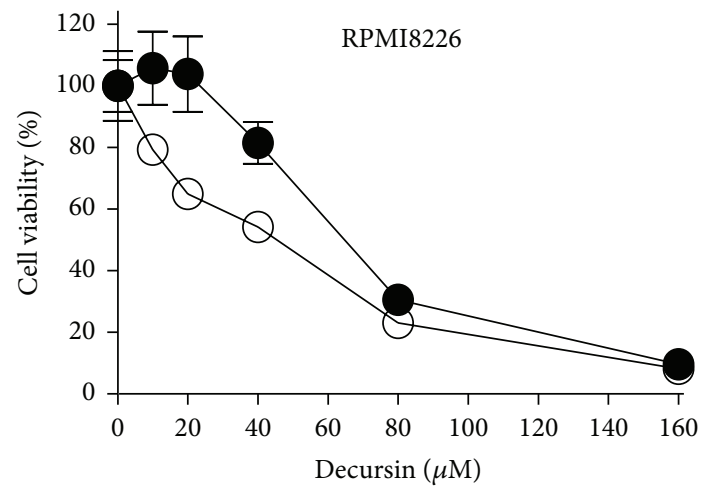

$24 h$
$-48 h$

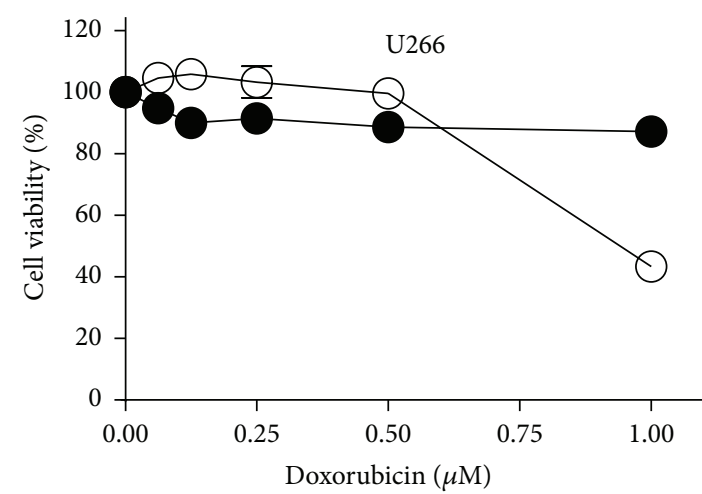

(b)

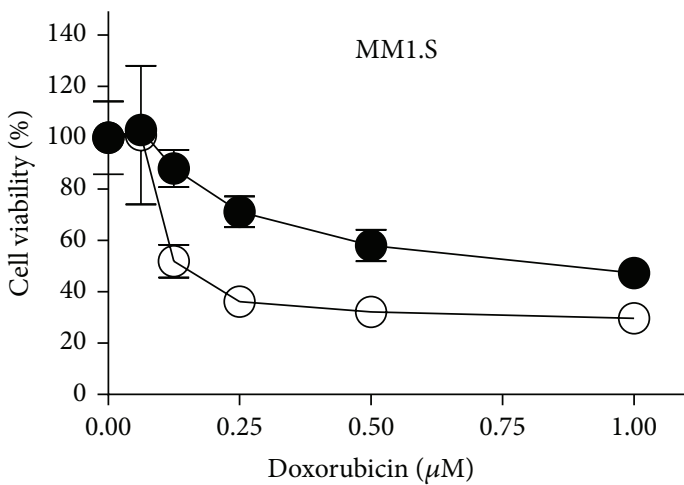

(d)

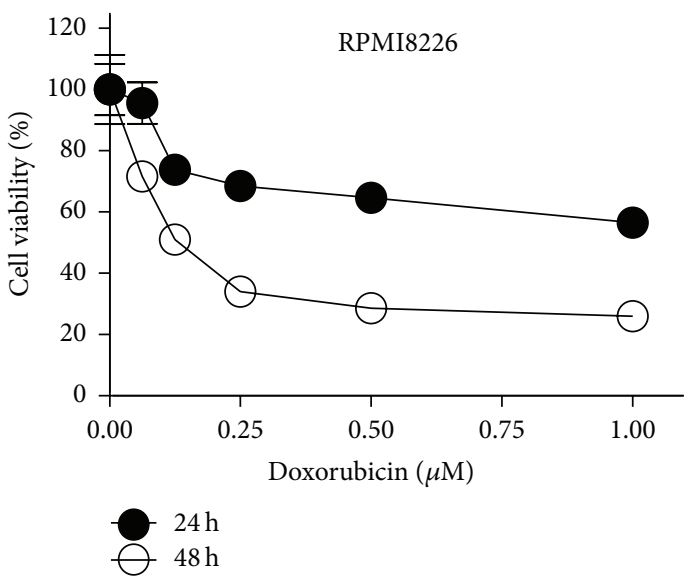

(f)

FIGURE 1: Cytotoxic effects of doxorubicin and decursin against multiple myeloma cells. (a) U266 cells were treated with various concentrations of decursin $(0,10,20,40,80$, or $160 \mu \mathrm{M})$ for $24 \mathrm{~h}$ or $48 \mathrm{~h}$. (b) U266 cells were treated with various concentrations of doxorubicin $(0,0.0625,0.125,0.25,0.5$, or $1 \mu \mathrm{M})$ for $24 \mathrm{~h}$ or $48 \mathrm{~h}$. (c) MM.1S cells were treated with various concentrations of decursin $(0,10,20,40,80$ or $160 \mu \mathrm{M})$ for 24 or $48 \mathrm{~h}$. (d) MM.1S cells were treated with various concentrations of doxorubicin $(0,0.0625,0.125,0.25,0.5$, or $1 \mu \mathrm{M})$ for 24 or $48 \mathrm{~h}$. (e) RPMI8226 cells were treated with various concentrations of decursin $(0,10,20,40$, 80, or $160 \mu \mathrm{M})$ for 24 or $48 \mathrm{~h}$. (f) RPMI8226 cells were treated with various concentrations of doxorubicin $(0,0.0625,0.125,0.25,0.5$, or $1 \mu \mathrm{M})$ for 24 or 48 h. Cytotoxicity of doxorubicin or decursin was evaluated by XTT assay. Data are presented as mean \pm SD for triplicate experiments.

were separated by electrophoresis on $4-12 \%$ NuPAGE BisTris gels. The proteins then was transferred to Hybond ECL transfer membrane and analyzed with anti-PARP, caspase-8, caspase- 9 , and caspase- 3 antibodies. Protein contents were normalized by reprobing the same membrane with anti- $\beta$ actin antibody (Sigma).
2.9. Measurement of Mitochondrial Membrane Potential. Mitochondrial potential was determined as previously described [27]. U266 cells treated with decursin and/or doxorubicin were incubated for $24 \mathrm{~h}$ at $37^{\circ} \mathrm{C}$ and harvested. After washing twice with cold PBS, the pellets were resuspended in $1 \mathrm{~mL}$ of $150 \mu \mathrm{M}$ TMRE and incubated for $30 \mathrm{~min}$ at $37^{\circ} \mathrm{C}$ in the 

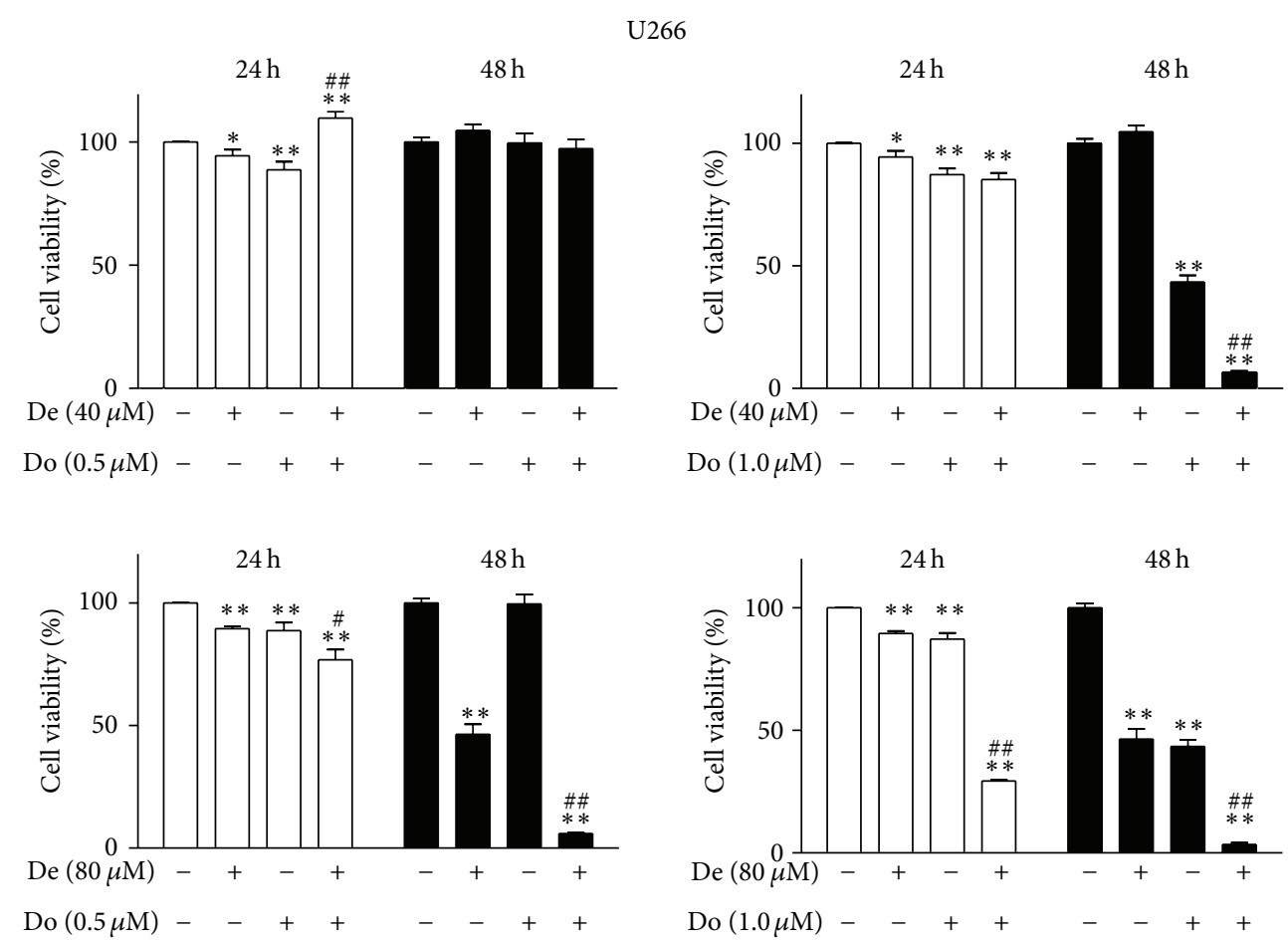

(a)

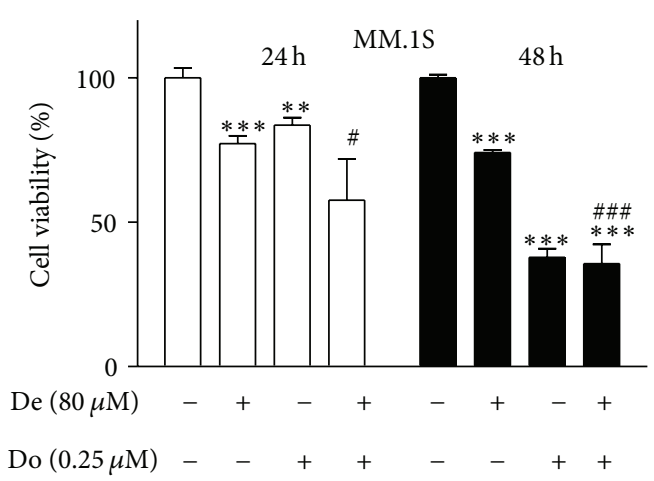

(b)

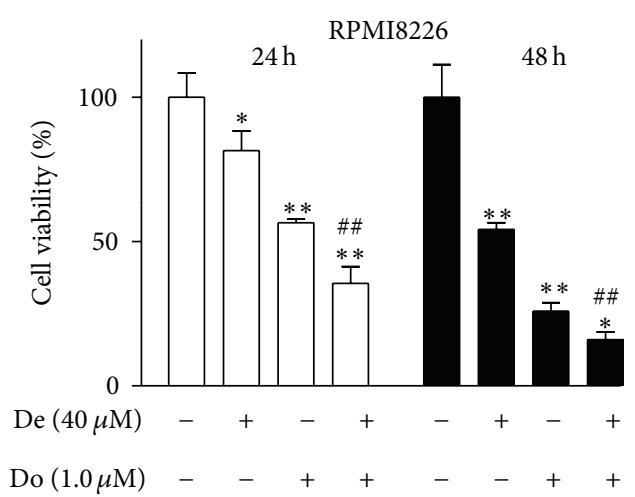

(c)

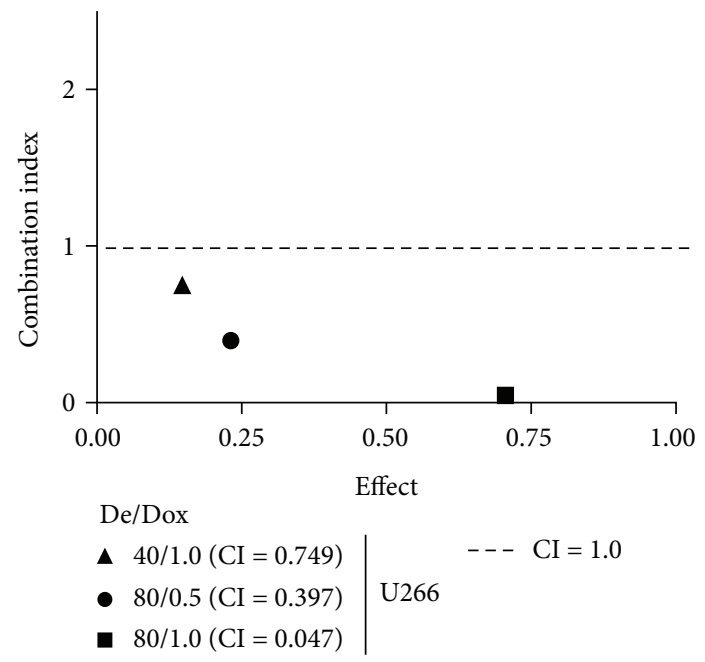

(d)

Figure 2: Continued. 


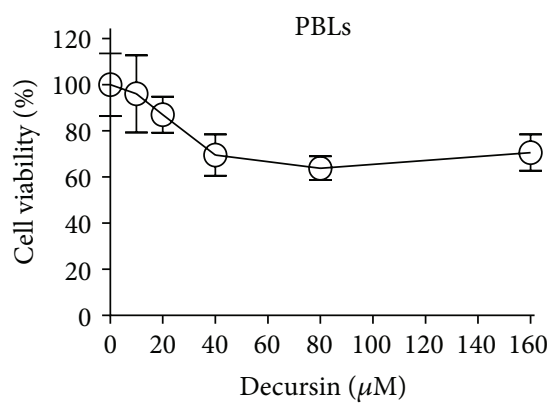

(e)
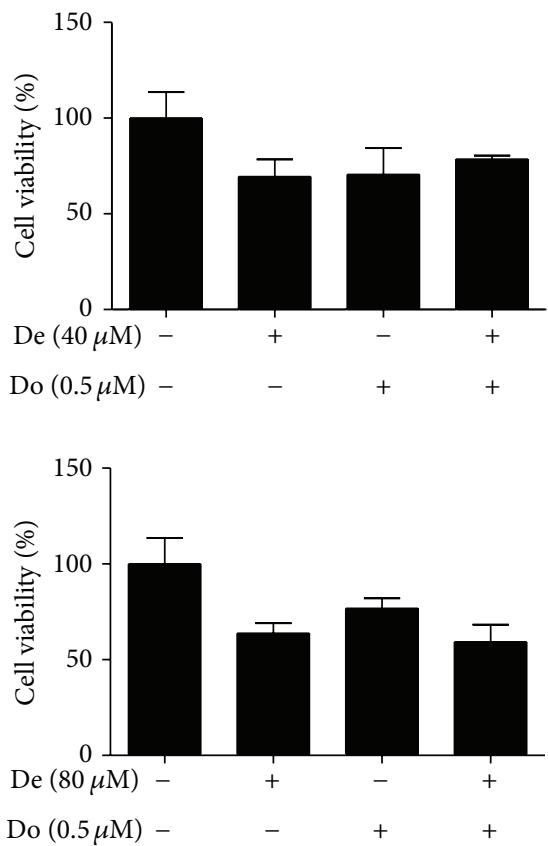

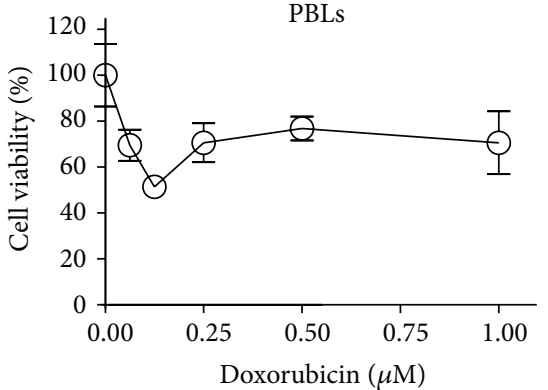

(f)
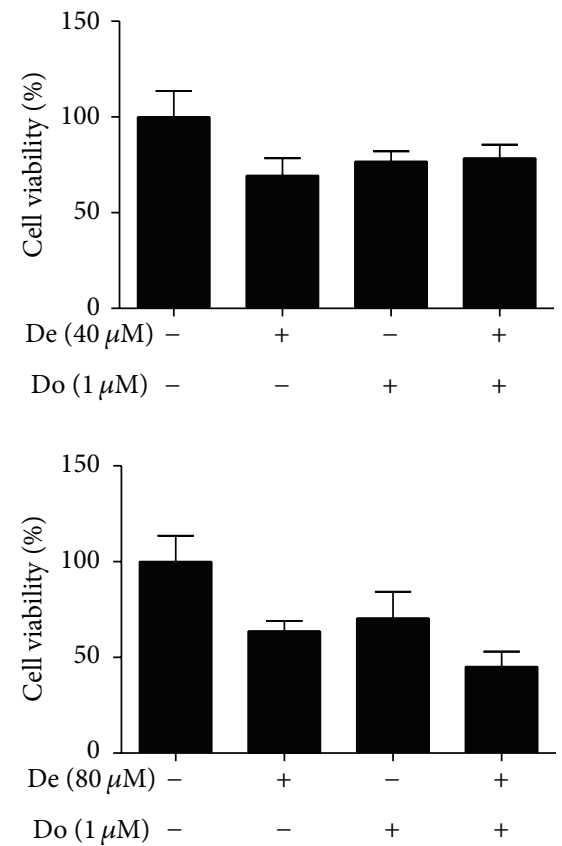

(g)

FIGURE 2: Synergistic effect of decursin and doxorubicin on the cytotoxicity in multiple myeloma cells. (a) U266 cells were treated with decursin $(40$ or $80 \mu \mathrm{M})$ and/or doxorubicin $(0.5$ or $1 \mu \mathrm{M})$ for 24 or $48 \mathrm{~h}$. (b) MM.1S cells were treated with decursin $(80 \mu \mathrm{M})$ and/or doxorubicin $(0.25 \mu \mathrm{M})$ for 24 or $48 \mathrm{~h}$. Cytotoxicity of doxorubicin and/or decursin was evaluated by XTT assay. (c) RPMI8226 cells were treated with decursin $(40 \mu \mathrm{M})$ and/or doxorubicin $(1 \mu \mathrm{M})$ for 24 or $48 \mathrm{~h}$. Data are presented as means \pm SD for triplicate experiments. ${ }^{*}, P<0.05,{ }^{* *} ; P<0.01$ and ${ }^{* * *} ; P<0.001$ versus untreated control. ${ }^{\#}, P<0.05,{ }^{\# \#} ; P<0.01,{ }^{\# \# \#}, P<0.001$ versus doxorubicin-treated cells. (d) The combination index (CI) between decursin and doxorubicin $(24 \mathrm{~h})$ was determined by the Chou-Talalay method and CalcuSyn software (Biosoft, Feruson, MO, USA) in U266 and MM.1S cells. Normal peripheral blood leukocytes (PBLs) were treated with various concentrations of (e) decursin $(10,20,40,80,160 \mu \mathrm{M})$ and/or (f) doxorubicin $(0,0.0625,0.125,0.25,0.5$ or $1 \mu \mathrm{M})$ for 24 . (g) Cytotoxicity of decursin $(40 \mu \mathrm{M}$ or $80 \mu \mathrm{M})$ and doxorubicin $(0.5$ or $1 \mu \mathrm{M})$ in PBLs was evaluated by XTT assay. Data are presented as mean \pm SD for triplicate experiments.

dark. The fluorescent intensities of cells were analyzed by flow cytometry (FACSCalibur, BD).

2.10. Statistical Analysis. All data were expressed as means \pm $\mathrm{SD}$ of three independent experiments. The statistically significant differences between untreated control and decur$\sin$ /doxorubicin treated groups were calculated by Student's $t$-test.

\section{Results}

3.1. Decursin and Doxorubicin Synergistically Enhanced the Cytotoxic Effect in Multiple Myeloma Cells. To evaluate the cytotoxic effect of decursin or doxorubicin, XTT assay was performed in human multiple myeloma (U266, MM.1S, and RPMI8226). Decursin did not influence the viability of U266 and MM1.S cells up to $80 \mu \mathrm{M}$ for $24 \mathrm{~h}$ culture (Figures 1(a) and 1(c)), while decursin showed significant cytotoxicity in all cells such as U266, MM1.S, and RPMI8226 cells at $80 \mu \mathrm{M}$ for $48 \mathrm{~h}$ culture (Figures 1(a), 1(c) and 1(e)). Doxorubicin at $1 \mu \mathrm{M}$ had a minimal cytotoxic effect for $24 \mathrm{~h}$ and decreased the viability only for $48 \mathrm{~h}$ culture in U266 cells (Figure 1(b)), while MM1.S and RPMI8226 cells were more sensitive to doxorubicin at $250 \mathrm{nM}$ than U266 cells after $48 \mathrm{~h}$ culture (Figures 1(d) and 1(f)). 

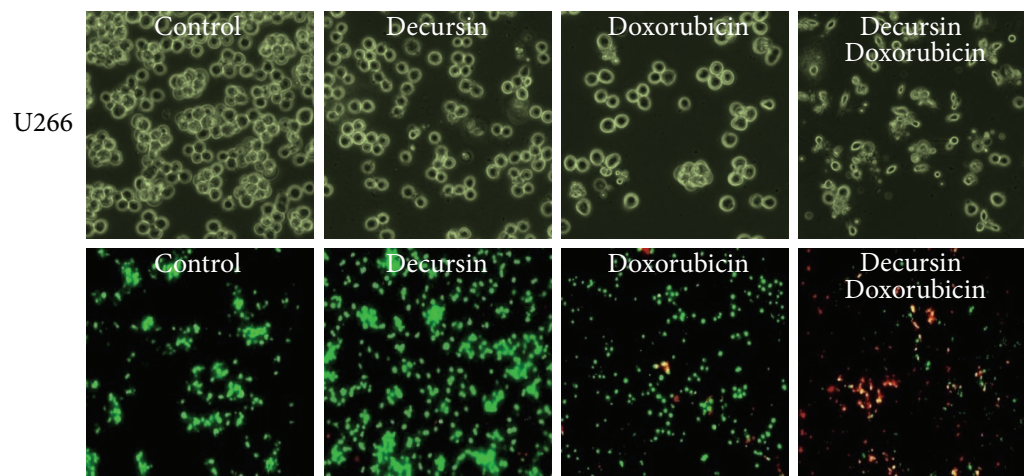

(a)
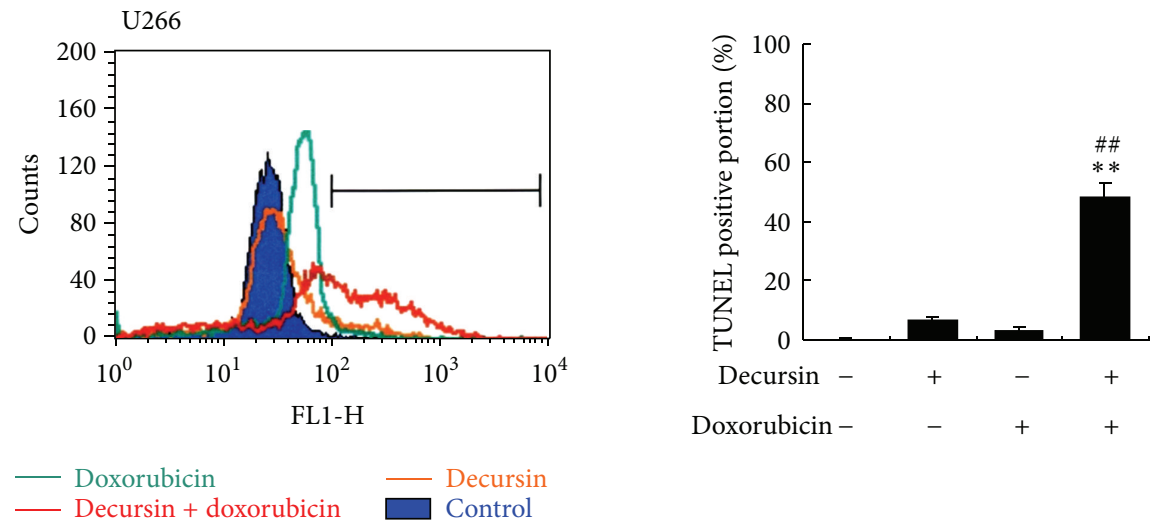

(b)

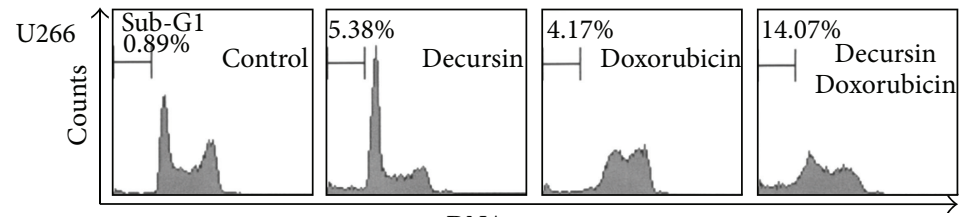

DNA contents
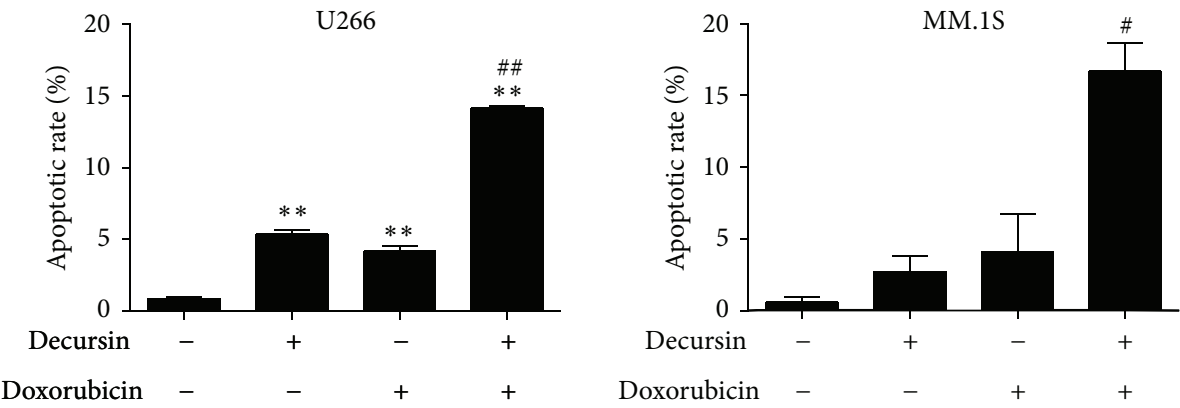

(c)

FIGURE 3: Effect of decursin on doxorubicin-induced apoptosis in multiple myeloma cells. U266 or MM.1S cells were treated with decursin $(80 \mu \mathrm{M})$ and/or doxorubicin $(1 \mu \mathrm{M})$ for $24 \mathrm{~h}$. (a) Top panel: morphological changes of U266 cells were observed under inverted microscope $(\times 200)$. Bottom panel: U266 cell death was determined by the calcein-AM-based live/dead assay as described in Section 2. Red highlights are dead cells, and green highlights live cells. Percent of dead cells was represented. (b) U266 cells were labelled with TdT-mediated dUTP nick end labeling (TUNEL) and analyzed by flow cytometry. (c) U266 or MM.1S cells were stained with PI after fixing in 75\% ethanol. DNA contents of sub-G1 were analyzed by flow cytometry. A representative result of three independent experiments is shown for each experiment. Data are presented as means \pm SD for triplicate experiments. ${ }^{*}, P<0.05$ and $^{* *}, P<0.01$ versus untreated control. ${ }^{\#}, P<0.05$ and $^{\# \#}, P<0.01$ versus doxorubicin-treated cells. 
To examine the synergistic activity of decursin and doxorubicin, U266 cells were treated with decursin (40 or $80 \mu \mathrm{M})$, doxorubicin $(0.5$ or $1 \mu \mathrm{M})$, or both for 24 or $48 \mathrm{~h}$. As shown in Figure $2(\mathrm{a})$, the cotreatment of decursin $(80 \mu \mathrm{M})$ and doxorubicin $(1 \mu \mathrm{M})$ for $24 \mathrm{~h}$ significantly decreased the viability $(71 \%)$ of $\mathrm{U} 266$ cells compared with that treated with doxorubicin (13\%) or decursin (11\%) alone (Figure 2(a)). A severe cytotoxic effect ( $>90 \%$ cell death) was observed in combination of decursin and doxorubicin $(40 \mu \mathrm{M} / 1 \mu \mathrm{M}$, $80 \mu \mathrm{M} / 0.5 \mu \mathrm{M}$ and $80 \mu \mathrm{M} / 1 \mu \mathrm{M}$ ) (Figure 2(a)). Likewise, cotreatment of decursin and doxorubicin $(80 \mu \mathrm{M} / 0.25 \mu \mathrm{M}$, and $40 \mu \mathrm{M} / 1 \mu \mathrm{M}$, resp.) enhanced the cytotoxicity in MM1.S and RPMI8226 cells (Figures 2(b) and 2(c)) with statistical significance using combination index (CI value = $0.749,0.397$, and 0.047 at $40 \mu \mathrm{M} / 1 \mu \mathrm{M}, 80 \mu \mathrm{M} / 0.5 \mu \mathrm{M}$, and $80 \mu \mathrm{M} / 1 \mu \mathrm{M}$, resp.) in U266 cells (Figure 2(d)). However, decursin and/or doxorubicin showed weak cytotoxicity against normal peripheral blood leukocytes (PBLs) (Figures 2(e), 2(f), and 2(g)).

\subsection{Doxorubicin and Decursin Drastically Induced Apoptosis} in Multiple Myeloma Cells. We observed after being exposed to decursin, doxorubicin, or both, some morphological changes of U266 cells were observed under a microscopy by live and dead assay (Figure 3(a)). The cotreated cells appeared to swell and with apoptotic shrinkage. To further confirm whether loss of the viability of the cells cotreated with decursin and doxorubicin was due to apoptosis, TUNEL, and live/dead assays were performed in U266 cells. The addition of decursin or doxorubicin alone had a minimal apoptotic effect on the cells. A similar result was obtained from TUNEL assay, in which the numbers of TUNEL-positive cells were significantly increased after the combination treatment (Figure 3(b)), while a few TUNEL-positive cells were detected after the addition of decursin or doxorubicin alone. Consistent with the above results, the co-treatment increased the population of sub-G1 DNA contents (14.07\%) compared to decursin $(5.38 \%)$ or doxorubicin $(4.17 \%)$ alone (Figure $3(\mathrm{c})$ ), suggesting that low doses of decursin and doxorubicin act in synergy for the induction of apoptosis in U266 cells. Similarly, in MM.1S cells, the cotreatment of decursin and doxorubicin remarkably induced apoptosis (sub-G1; 16.67\%) compared to decursin $(3.2 \%)$ or doxorubicin $(4.8 \%)$ alone.

3.3. Doxorubicin and Decursin Induced Mitochondria-Dependent Apoptosis in Multiple Myeloma Cells. Mitochondria plays a crucial role in the regulation of the induction of caspase-dependent and -independent apoptosis [28]. Thus, we examined whether apoptosis induced by decursin plus doxorubicin is mediated through caspase activation. The cotreatment induced a high level of cleaved caspase-3, an effector caspase, PARP (a substrate of caspase-3), compared with that treated with decursin or doxorubicin alone in U266 cells, MM1.S and RPMI8226 cells (Figures 4(a) and 4(b)). Furthermore, cleavage of caspase- 9 was observed in three multiple myeloma cells by the combination of decursin and doxorubicin in a time-dependent manner in U266 cells (Figure 4(c)). Consistently, the apoptosis induction was blocked in pretreatment with caspase- 9 inhibitor, but not caspase- 8 inhibitor (data not shown). These results suggest that the combination of decursin and doxorubicin induces apoptosis through mitochondria-dependent pathway.

The mitochondria membrane potential (MMP), an important parameter of mitochondrial function, was measured by flow cytometry in cells treated with decursin, doxorubicin, or both. The cotreatment significantly reduced fluorescence intensity (from $94.09 \%$ to $65.86 \%$ in U266 cells, and from $93.92 \%$ to $65.35 \%$ in MM.1S cells, implying the loss of MMP (Figure 4(d)), while either drug alone had no effect on the MMP. These results suggest that apoptosis induced by the concurrent treatment of decursin and doxorubicin is through the change of the membrane potential of the mitochondria in U266 and MM.1S cells.

\subsection{Doxorubicin and Decursin Targeted Multiple Signaling} Molecules in Multiple Myeloma Cells. Next, we investigated whether STAT3 signaling is involved in the synergistic regulation of multiple myeloma cell survival by the cotreatment of decursin and doxorubicin. As expected, the combined treatment significantly inhibited the level of phospho-STAT3, compared to decursin or doxorubicin alone (Figure 5(a)). STAT3 is activated by the upstream kinases JAK or Src family [29] and can regulate oncogenesis by targeting various gene products [30]. Using immunoblot analysis, we showed that the combination treatment of decursin and doxorubicin dramatically suppressed the level of phospho-JAK2 and phospho-Src, compared to either drug alone (Figure 5(b)). The combination treatment also increased the magnitudes of decursin- or doxorubicin-mediated downregulation of cyclin D1 and survivin that are the products of STAT3 target genes (Figure 5(c)). The effects of decursin and doxorubicin on STAT3-related signaling molecules were also shown in cells treated for 8,16 , or $24 \mathrm{~h}$ (Figure $5(\mathrm{~d})$ ). As shown in Figure 5(d), the combination treatment suppressed pJAK2, pSTAT3, and Cyclin D1 and activated SHP-2 in a time-dependent manner in U266 cells, indicating that the synergistic effect on JAK2-STAT3-Cyclin D1 signal axis in STAT3-positive U266 cells. Conversely, the broadly acting tyrosine phosphatases inhibitor pervanadate reversed STAT3 inactivation and also PARP cleavage and caspase- 3 activation induced by combined treatment of doxorubicin and decursin in U266 cells, indicating the important role of STAT3 in apoptosis induced by the combination of doxorubicin and decursin in STAT3 active U266 cells.

To explore whether the combination effect of decursin and doxorubicin is directly regulated by STAT3 signaling, parallel experiments were performed in MM.1S (STAT3 inactive) cells. As shown in Figure 5(e), the cotreatment of decursin and doxorubicin also suppressed the levels of phospho-JAK2 and cyclin D1 in MM.1S cells. However, phosphorylation of STAT3 was not observed in MM.1S cells (data not shown). In addition, to elucidate whether decursin suppression of STAT3 is associated with decursin and doxorubicin-induced apoptosis, U266 cells cotreated with decursin and doxorubicin were treated with pervanadate and expressions of SHP2, p-STAT3, PARP, and caspase-3 were then analyzed. Pervanadate treatment resulted in an 


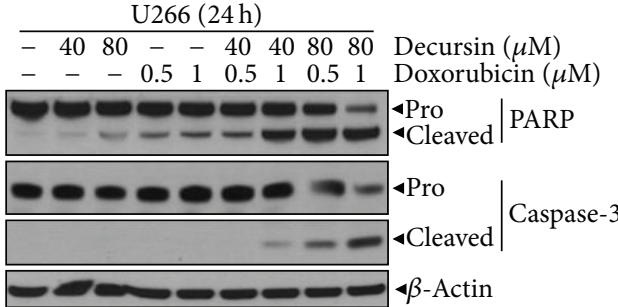

(a)
MM.1S (24h)

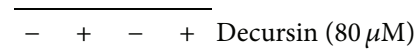

$-\quad+\quad+$ Doxorubicin $(0.25 \mu \mathrm{M})$

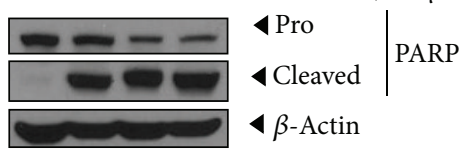

RPMI $8226(24 \mathrm{~h})$

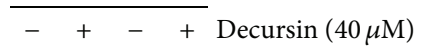

$-\quad+\quad+\quad$ Doxorubicin $(1 \mu \mathrm{M})$

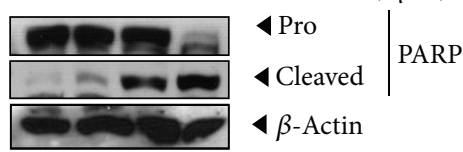

(b)

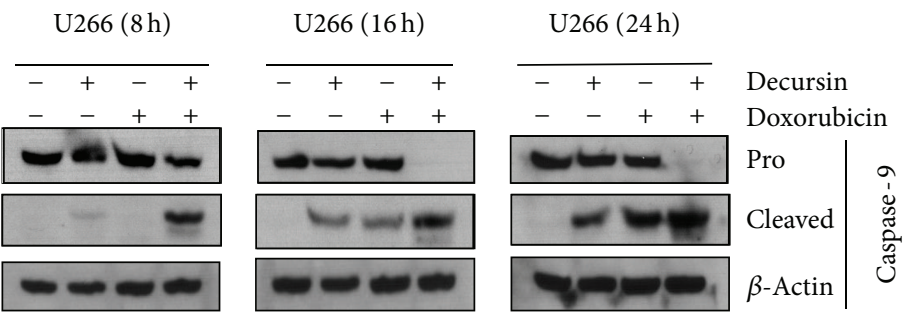

(c)
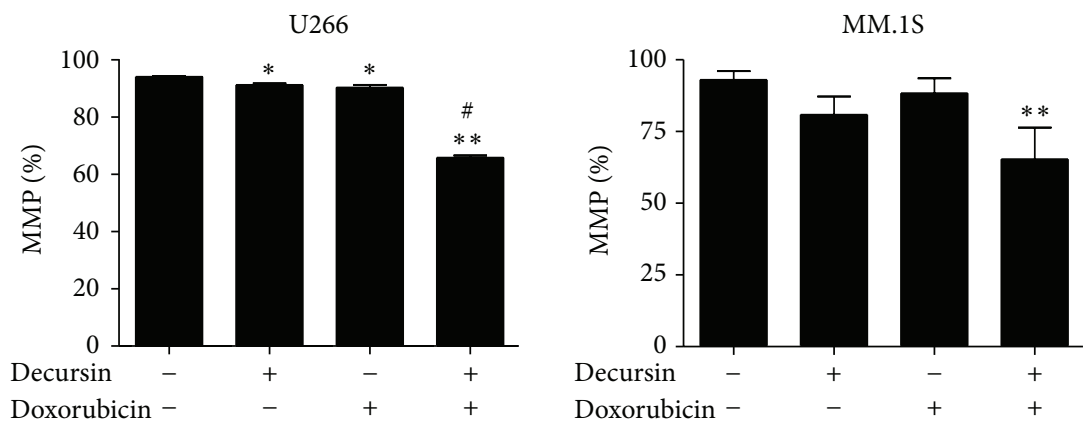

(d)

FIGURE 4: Effect of decursin on mitochondria-dependent apoptosis in doxorubicin-treated multiple myeloma cells. (a) U266 cells were treated with decursin $(40$ or $80 \mu \mathrm{M})$ and/or doxorubicin $(0.5$ or $1 \mu \mathrm{M})$ for $24 \mathrm{~h}$ and subjected to western blotting for PARP and caspase-3. (b) MM.1S and RPMI8226 cells were treated with decursin $(40,80 \mu \mathrm{M}$, resp.) and/or doxorubicin $(0.25,1 \mu \mathrm{M}$, resp.) for $24 \mathrm{~h}$. Western blotting was performed for PARP. (c) U266 cells were treated with decursin $(80 \mu \mathrm{M})$ and/or doxorubicin $(1 \mu \mathrm{M})$ for 8 or $16 \mathrm{~h}$, and with decursin $(40$ or $80 \mu \mathrm{M})$ and/or doxorubicin $(0.5$ or $1 \mu \mathrm{M})$ for $24 \mathrm{~h}$. Western blotting was performed for caspase-9. (d) U266 or MM.1S cells were treated with decursin $(80 \mu \mathrm{M})$ and/or doxorubicin $(0.5$ or $1 \mu \mathrm{M})$ for $24 \mathrm{~h}$ and stained with tetramethylrhodamine ethyl ester (TMRE) for $30 \mathrm{~min}$ at $37^{\circ} \mathrm{C}$, and mitochondrial membrane potential (MMP) was detected by flow cytometry. Graphs represent the percentages of MMP. Data are presented as means $\pm \mathrm{SD}$ for triplicate experiments. ${ }^{*}, P<0.05$ and $^{* *}, P<0.01$ versus untreated control. ${ }^{*}, P<0.05$, versus doxorubicin-treated cells.

increase of p-STAT3 and clearly blocked PARP cleavage, caspase- 3 activation, and SHP-2 in combination of decursin and doxorubicin suggesting that decursin and doxorubicininduced apoptosis via STAT3 inactivation in U266 cells (Figure 6(a)).

Since the combination of decursin- and doxorubicininduced apoptosis in three multiple myeloma cells regardless of STAT3 existence, we examined another signaling pathway relevant to synergistic antitumor effect of combination of decursin and doxorubicin in three multiple myeloma cells. As shown in Figure 6(b), the combination of decursin and doxorubicin downregulated the phosphorylation of p-mTOR and its downstream $\mathrm{S} 6 \mathrm{~K} 1$ in the three multiple myeloma cells but did not affect PI3 K and Akt signaling (data not shown). Also, the combination of decursin and doxorubicin upregulated the phosphorylation of extracellular signal-regulated kinase (ERK) but did not affect p38 and JNK (data not shown) in the three multiple myeloma cells.

\section{Discussion}

Drug resistance is a severe problem in treating multiple myeloma, a hematologic malignant disorder [31]. Overexpression of p-glycoprotein (p-gp) [32] and the multi-drugrelated protein (MRP) [33] are ones of many possible mechanisms of drug resistance in cancer therapy. The molecules allow exporting or excluding anticancer drugs, resulting in drug resistance. Various chemosensitizers or resistance modifiers such as anthracyclin (doxorubicin), vinca alkaloids (vincristine and vinblastine), and epipodophyllotoxins are chemotherapeutics that are affected by the drug efflux pump p-glycoprotein. Cell-cell and cell-stroma interactions using various adhesion molecules including very late antigen-4 (VLA-4), vascular adhesion molecule (VCAM), leukocyte function-associated antigen 1 (LFA-1), and intercellular adhesion molecule-1 (ICAM-1) [34] are important throughout myeloma pathogenesis and also contribute to drug resistance 


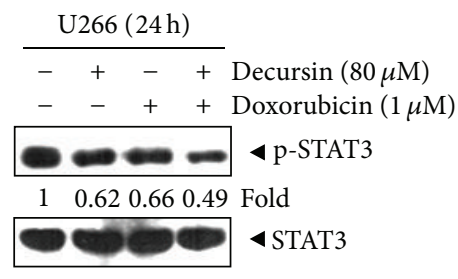

(a)

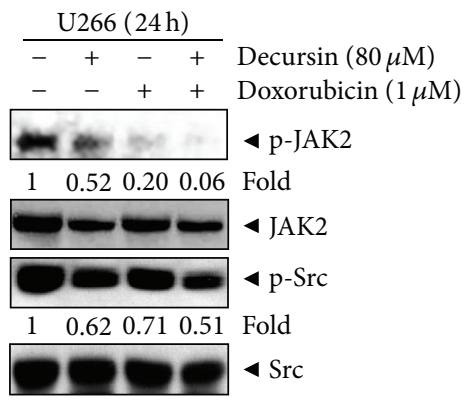

(b)

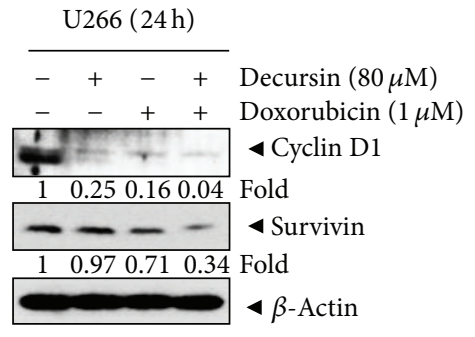

(c)
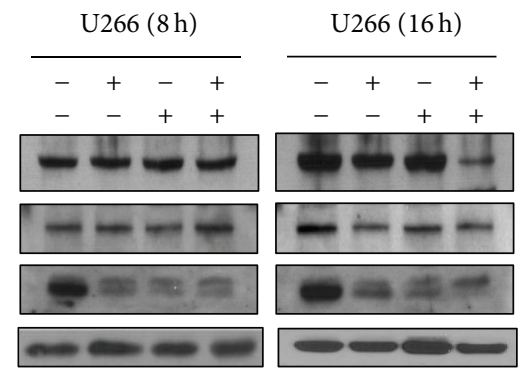

$\mathrm{U} 266(24 \mathrm{~h})$

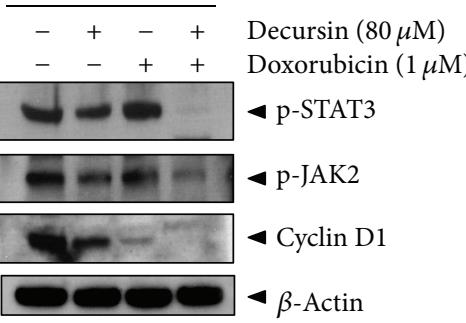

(d)

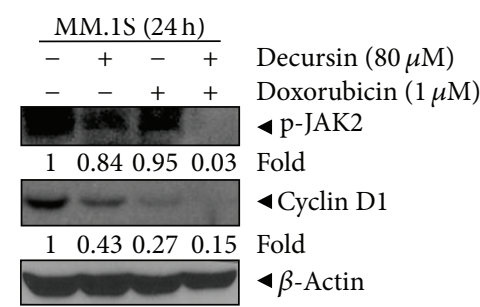

(e)

FIGURE 5: Effect of doxorubicin and decursin on STAT3 signaling in multiple myeloma cells. (a-c) U266 cells were treated with decursin $(80 \mu \mathrm{M})$ and/or doxorubicin $(1 \mu \mathrm{M})$ for $24 \mathrm{~h}$. Cell lysates were prepared and subjected to western blotting for (a) phospho-STAT3 and STAT3, phospho-JAK2, JAK2, phospho-Src (b) and Src, and (c) cyclin D1 and survivin. (d) U266 cells were treated with decursin (80 $\mu$ M) and/or doxorubicin $(1 \mu \mathrm{M})$ for 8,16 , or $24 \mathrm{~h}$. Western blotting was performed for phospho-STAT3, phospho-JAK2, and cyclin D1. (e) MM.1S cells were treated with decursin $(80 \mu \mathrm{M})$ and/or doxorubicin $(1 \mu \mathrm{M})$ for $24 \mathrm{~h}$. Western blotting was performed for phospho-JAK2 and cyclin D1. A representative result of three independent experiments is shown for each experiment.

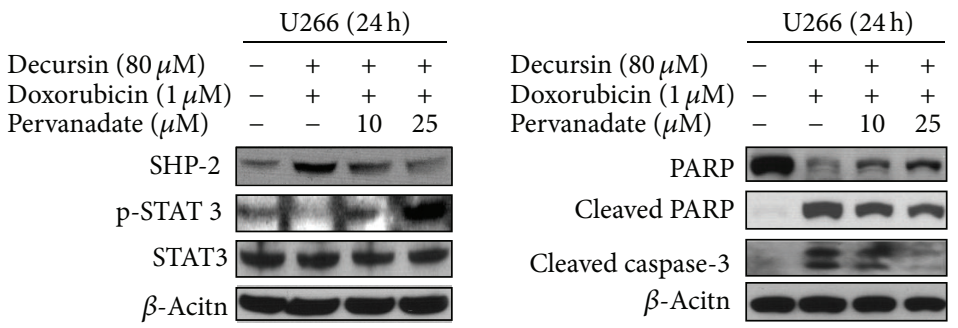

(a)
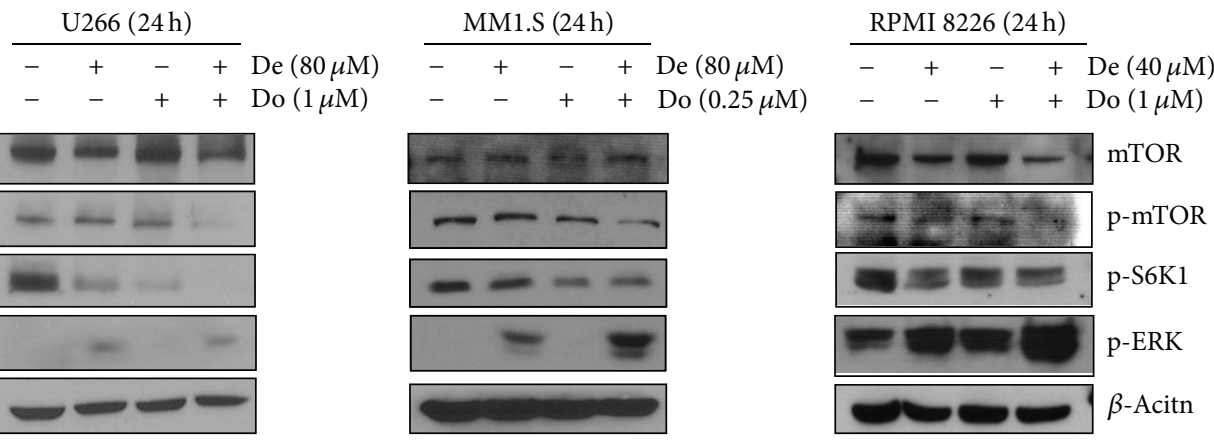

(b)

FIGURE 6: Effect of doxorubicin and decursin on mTOR pathway in multiple myeloma cells. (a) Effect of pervanadate on the STAT3 inactivation, SHP-2 activation, and cleavages of caspase-3 and PARP induced by combination of decursin and doxorubicin in U266 cells. (b) Effect of combination of decursin and doxorubicin on the phosphorylation of mTOR, S6 K1, and ERK in U266, MM1.S, and RPMI8226 cells. The cells were treated with decursin and/or doxorubicin for $24 \mathrm{~h}$. Western blotting was performed with antibodies of mTOR, phosphomTOR, phospho-S6 K1, phospho-ERK, and $\beta$-actin. 
although multiple myeloma patients are initially responsive to these drugs.

The influence on the susceptibility to apoptosis is another mechanism of drug resistance to chemo or radiotherapy [35]. Several different combination regimens have been applied for the treatment of multiple myeloma. Especially, VAD regimen (vincristine $(\mathrm{V})+$ doxorubicin $(\mathrm{A})+$ dexamethasone (D)) is known as an efficient treatment that induces a more rapid response than other regimens for multiple myeloma $[36,37]$. However, serious side effects such as myelotoxicity, neurotoxicity, and nausea still remain problematic for multiple myeloma treatment [38]. Thus, modified or novel combination regimen(s) will be necessary to improve the tolerability and efficacy of multiple myeloma therapy. For this purpose, natural compounds are ideal materials for developing new combination regimens for multiple myeloma, since most of these compounds are less or non toxic to normal cells, but able to target cancer cells specifically. For example, curcumin in combination with bortezomib synergistically induced apoptosis in human multiple myeloma U266 cells [39]. Capsaicin also significantly stimulated the apoptotic effects of Velcade and thalidomide in multiple myeloma cells [40].

In this regards, we investigated that decursin synergistically augmented apoptosis induction in its combination with doxorubicin, a component of VAD regimen, in U266 multiple myeloma cells. Several previous papers reported decursininduced apoptosis in cancer cells. Decursin inhibited growth of human bladder and colon cancer cells via the induction of apoptosis, of G1-phase arrest, and activation of extracellular signal-regulated kinase [13]. Decursin suppressed human androgen-independent PC3 prostate cancer cell proliferation by promoting the degradation of beta-catenin [14]. We recently found that decursin-mediated apoptosis via inhibition of cyclooxygenase-2- (COX-2-) dependent survivin expression in human myeloid leukemia cells [12].

In the present study, we found that the combined treatment of decursin and doxorubicin synergistically elevated levels of the magnitude of apoptosis in human multiple myeloma cells, while it shows weak cytotoxicity in normal peripheral blood leukocytes (PBLs). The results obtained from cell viability, TUNEL, and live/dead assays were in a good agreement, suggesting that the cotreatment is able to increase the anticancer activity against multiple myeloma cells. In this apoptotic process, caspase family, aspartatespecific cysteine proteases, played a central role [41]. Activation of caspase-3 and cleavage of its substrates such as PARP and lamin A are the hallmarks of apoptosis [42]. In our results, the combination treatment augmented the levels of the active forms of caspase-3 and -9 , as well as of PARP cleavage. In addition, the combination treatment drastically induced the loss of MMP. Therefore, the results further confirmed the synergy of decursin and doxorubicin in the induction of apoptosis in multiple myleoma cells.

STAT is a family of six different transcription factors that play crucial roles in cytokine signaling [43]. STAT3 is often constitutively activated in various types of human cancer including multiple myeloma and closely associated with cancer cell proliferation and antiapoptosis [44-46]. It has been well known that the growth of multiple myeloma cells is regulated by signal transduction through the JAK/STAT pathway [24]. Thus, STAT3 has been implicated as a potential therapeutic target for multiple human cancer [47]. Kim and colleagues recently reported that decursin antagonized STAT3 signaling for the sensitization of U266 cells to apoptosis [17]. In the present study, combination of decursin and doxorubicin suppressed the phosphorylation of JAK2 and Src, which are upstream protein tyrosine kinases of the STAT pathway, and STAT3 in STAT3-positive U266 cells. There are evidences that STAT3 activation is negatively regulated by protein tyrosine phosphatases (PTPs) including SHP-1, SHP-2, PTEN, PTP- $\varepsilon$, and SOCS-1 [48, 49]. Here, the combination of decursin and doxorubicin activated SHP-2 in U266 cells. Conversely, pervanadate (a general PTP inhibitor) significantly reversed STAT3 inactivation induced by combination of decursin and doxorubicin in U266 cells, supporting an important role of the PTP in dephosphorylation of STAT3 in U266 cells. Also, we found that the cotreatment of decursin and doxorubicin synergistically reduced mitochondrial membrane potential and attenuated the expression of cyclind-D1 in U266 cells, while the combination treatment downregulated the phosphorylation of JAK2 and attenuated the expression of cyclinD1 in STAT3 inactive MM1S cells, implying STAT3 independent pathway.

Given that the combination of decursin and doxorubicin induced cytotoxicity or apoptosis in three multiple myeloma cells regardless of STAT3 existence, another signaling pathway can be involved in the synergistic antitumor effect of combination of decursin and doxorubicin in three multiple myeloma cells. The mTOR regulates the various cellular processes such as cell growth, angiogenesis, and survival [50] through the phosphorylation of P70S6 K [51]. Also, mTOR regulates the cell proliferation through controlling the production of cyclin D1 [52] Thus, several clinical studies revealed that inhibition of mTOR might be a good target for cancer therapy $[20,53]$ and several drugs such as everolimus, ridaforolimus, and temsirolimus as mTOR inhibitors have been used for clinical trials [54-56]. In the current study, the phosphorylation of mTOR and S6K1 was inhibited by combination of decursin and doxorubicin in three multiple myeloma cells, implying that combination of decursin and doxorubicin induces apoptosis via mTOR/S6K1 pathway. Also, with the evidence that loss of mTOR resulted in an activation of ERK [57], the combination of decursin and doxorubicin upregulated the phosphorylation of ERK in the three multiple myeloma cells, indicating that ERK activation is associated with inhibition of mTOR/S6K1 signaling.

Anticancer activity of doxorubicin has been explained by inhibiting DNA polymerase [58] and topoisomerase II [59]. Spatial or temporal distribution of the enzymes has been reported during cancer cell proliferation [60, 61]. Indeed, our FACS analysis data using PI staining showed antiproliferation activity of decursin and doxorubicin. Similarly, several studies reported that anti-cancer activity of doxorubicin was significantly increased in combination of other drugs such as all-trans retinoid (ATR) [62] and artesunate [63]. It can be assumed that synergistic anticancer effect of decursin and doxorubicin may result partially from 
inhibiting DNA polymerase or topoisomerase II activities in addition to the suppression of STAT3 activation. Thus, additional experiments are required to verify this hypothesis in the near future.

\section{Conclusions}

In summary, our results demonstrate the synergistic effect of decursin and doxorubicin on the induction of apoptosis via the inhibition of mTOR and/or STAT3 signaling pathway in multiple myeloma cells. Notably, the synergy of decursin and doxorubicin was verified by calculating $\mathrm{CI}$ value $(\mathrm{CI}<1)$. Thus, our findings propose that the combination of decursin and doxorubicin may be beneficial for the improvement of the treatment of multiple myeloma patients.

\section{Conflict of Interests}

The authors have declared that no conflict of interests exists.

\section{Authors' Contribution}

Jinsil Jang, Soo-Jin Jeong, Hee-Young Kwon, Ji Hoon Jung, and Eun Jung Sohn contributed equally to this work and should be considered co-first authors.

\section{Acknowledgment}

This work was supported by the National Research Foundation of Korea (NRF) Grant funded by the Korea government (MEST) (no. 2012-0005755).

\section{References}

[1] R. Feinman, J. Koury, M. Thames, B. Barlogie, J. Epstein, and D. S. Siegel, "Role of NF- $\kappa$ B in the rescue of multiple myeloma cells from glucocorticoid-induced apoptosis by bcl-2," Blood, vol. 93, no. 9, pp. 3044-3052, 1999.

[2] H. Brenner, A. Gondos, and D. Pulte, "Recent major improvement in long-term survival of younger patients with multiple myeloma," Blood, vol. 111, no. 5, pp. 2521-2526, 2008.

[3] Haematology, UmfBCfSi, "Diagnosis and management of multiple myeloma," The British Journal of Haematology, vol. 115, no. 3, pp. 522-540, 2001.

[4] T. Hideshima, C. Mitsiades, G. Tonon, P. G. Richardson, and K. C. Anderson, "Understanding multiple myeloma pathogenesis in the bone marrow to identify new therapeutic targets," Nature Reviews Cancer, vol. 7, no. 8, pp. 585-598, 2007.

[5] S. V. Rajkumar, R. Fonseca, A. Dispenzieri et al., "Thalidomide in the treatment of relapsed multiple myeloma," Mayo Clinic Proceedings, vol. 75, no. 9, pp. 897-901, 2000.

[6] P. G. Richardson, B. Barlogie, J. Berenson et al., "A phase 2 study of Bortezomib in relapsed, refractory myeloma," The New England Journal of Medicine, vol. 348, no. 26, pp. 2609-2617, 2003.

[7] P. G. Richardson, E. Blood, C. S. Mitsiades et al., "A randomized phase 2 study of lenalidomide therapy for patients with relapsed or relapsed and refractory multiple myeloma," Blood, vol. 108, no. 10, pp. 3458-3464, 2006.
[8] R. Stein, M. R. Smith, S. Chen, M. Zalath, and D. M. Goldenberg, "Combining milatuzumab with bortezomib, doxorubicin, or dexamethasone improves responses in multiple myeloma cell lines," Clinical Cancer Research, vol. 15, no. 8, pp. 2808-2817, 2009.

[9] D. Chauhan, L. Catley, T. Hideshima et al., "2-methoxyestradiol overcomes drug resistance in multiple myeloma cells," Blood, vol. 100, no. 6, pp. 2187-2194, 2002.

[10] E. Sanchez, M. Li, J. A. Steinberg et al., "The proteasome inhibitor CEP-18770 enhances the anti-myeloma activity of bortezomib and melphalan," The British Journal of Haematology, vol. 148, no. 4, pp. 569-581, 2010.

[11] H. J. Chi and H. S. Kim, "Studies on the components of Umbelliferae plants in Korea: pharmacological study of decursin, decursinol and nodakenin," Korean Journal of Pharmacognosy, vol. 1, pp. 25-32, 1970.

[12] Q. Ahn, S. J. Jeong, H. J. Lee et al., "Inhibition of cyclooxygenase-2-dependent survivin mediates decursininduced apoptosis in human KBM-5 myeloid leukemia cells," Cancer Letters, vol. 298, no. 2, pp. 212-221, 2010.

[13] W. J. Kim, S. J. Lee, Y. D. Choi, and S. K. Moon, "Decursin inhibits growth of human bladder and colon cancer cells via apoptosis, G1-phase cell cycle arrest and extracellular signalregulated kinase activation," International Journal of Molecular Medicine, vol. 25, no. 4, pp. 635-641, 2010.

[14] G. Y. Song, J. H. Lee, M. Cho, B. S. Park, D. E. Kim, and S. $\mathrm{Oh}$, "Decursin suppresses human androgen-independent PC3 prostate cancer cell proliferation by promoting the degradation of $\beta$-catenin," Molecular Pharmacology, vol. 72, no. 6, pp. 15991606, 2007.

[15] J. H. Kim, S. J. Jeong, H. Y. Kwon et al., "Decursin prevents cisplatin-induced apoptosis via the enhancement of antioxidant enzymes in human renal epithelial cells," Biological and Pharmaceutical Bulletin, vol. 33, no. 8, pp. 1279-1284, 2010.

[16] L. Li, W. Li, S. W. Jung, Y. W. Lee, and Y. H. Kim, "Protective effects of decursin and decursinol angelate against amyloid $\beta$ protein-induced oxidative stress in the PC12 cell line: the role of Nrf2 and antioxidant enzymes," Bioscience, Biotechnology and Biochemistry, vol. 75, no. 3, pp. 434-442, 2011.

[17] H. J. Kim, S. M. Kim, K. R. Park et al., "Decursin chemosensitizes human multiple myeloma cells through inhibition of STAT3 signaling pathway," Cancer Letters, vol. 301, no. 1, pp. 2937, 2011.

[18] H. Hosoi, M. B. Dilling, T. Shikata et al., "Rapamycin causes poorly reversible inhibition of mTOR and induces p53independent apoptosis in human rhabdomyosarcoma cells," Cancer Research, vol. 59, no. 4, pp. 886-894, 1999.

[19] A. R. Tee and C. G. Proud, "Staurosporine inhibits phosphorylation of translational regulators linked to mTOR," Cell Death and Differentiation, vol. 8, no. 8, pp. 841-849, 2001.

[20] R. Li, R. Wang, R. Zhai, and Z. Dong, "Targeted inhibition of mammalian target of rapamycin (mTOR) signaling pathway inhibits proliferation and induces apoptosis of laryngeal carcinoma cells in vitro," Tumori, vol. 97, no. 6, pp. 781-786, 2011.

[21] C. Jiang, H. J. Lee, G. X. Li et al., "Potent antiandrogen and androgen receptor activities of an Angelica gigas-containing herbal formulation: identification of decursin as a novel and active compound with implications for prevention and treatment of prostate cancer," Cancer Research, vol. 66, no. 1, pp. 453463, 2006.

[22] S. Lee, Y. S. Lee, S. H. Jung, K. H. Shin, B. K. Kim, and S. S. Kang, "Anti-tumor activities of decursinol angelate and decursin from 
Angelica gigas," Archives of Pharmacal Research, vol. 26, no. 9, pp. 727-730, 2003.

[23] K. Nilsson, H. Bennich, S. G. Johansson, and J. Pontén, "Established immunoglobulin producing myeloma (IgE) and lymphoblastoid (IgG) cell lines from an IgE myeloma patient," Clinical and Experimental Immunology, vol. 7, no. 4, pp. 477489, 1970.

[24] S. Greenstein, N. L. Krett, Y. Kurosawa et al., "Characterization of the MM.1 human multiple myeloma (MM) cell lines: a model system to elucidate the characteristics, behavior, and signaling of steroid-sensitive and -resistant MM cells," Experimental Hematology, vol. 31, no. 4, pp. 271-282, 2003.

[25] L. Zhao, J. L. Au, and M. G. Wientjes, "Comparison of methods for evaluating drug-drug interaction," Frontiers in Bioscience (Elite edition), vol. 2, pp. 241-249, 2010.

[26] M. Herrmann, H. M. Lorenz, R. Voll, M. Grunke, W. Woith, and J. R. Kalden, "A rapid and simple method for the isolation of apoptotic DNA fragments," Nucleic Acids Research, vol. 22, no. 24, pp. 5506-5507, 1994.

[27] M. Mancini, B. O. Anderson, E. Caldwell, M. Sedghinasab, P. B. Paty, and D. M. Hockenbery, "Mitochondrial proliferation and paradoxical membrane depolarization during terminal differentiation and apoptosis in a human colon carcinoma cell line," Journal of Cell Biology, vol. 138, no. 2, pp. 449-469, 1997.

[28] L. A. Pradelli, M. Bénéteau, and J. E. Ricci, "Mitochondrial control of caspase-dependent and -independent cell death," Cellular and Molecular Life Sciences, vol. 67, no. 10, pp. 15891597, 2010.

[29] S. J. Schreiner, A. P. Schiavone, and T. E. Smithgall, "Activation of STAT3 by the Src family kinase Hck requires a functional SH3 domain," The Journal of Biological Chemistry, vol. 277, no. 47, pp. 45680-45687, 2002.

[30] R. Buettner, L. B. Mora, and R. Jove, "Activated STAT signaling in human tumors provides novel molecular targets for therapeutic intervention," Clinical Cancer Research, vol. 8, no. 4, pp. 945-954, 2002.

[31] W. S. Dalton, T. M. Grogan, P. S. Meltzer et al., "Drug-resistance in multiple myeloma and non-Hodgkin's lymphoma: detection of P-glycoprotein and potential circumvention by addition of verapamil to chemotherapy," Journal of Clinical Oncology, vol. 7, no. 4, pp. 415-424, 1989.

[32] J. A. Endicott and V. Ling, "The biochemistry of P-glycoproteinmediated multidrug resistance," Annual Review of Biochemistry, vol. 58, pp. 137-171, 1989.

[33] M. R. Abbaszadegan, B. W. Futscher, W. T. Klimecki, A. List, and W. S. Dalton, "Analysis of multidrug resistance-associated protein (MRP) messenger RNA in normal and malignant hematopoietic cells," Cancer Research, vol. 54, no. 17, pp. 46764679, 1994.

[34] R. Schmidmaier, K. Mörsdorf, P. Baumann, B. Emmerich, and G. Meinhardt, "Evidence for cell adhesion-mediated drug resistance of multiple myeloma cells in vivo," International Journal of Biological Markers, vol. 21, no. 4, pp. 218-222, 2006.

[35] W. R. Sellers and D. E. Fisher, "Apoptosis and cancer drug targeting," Journal of Clinical Investigation, vol. 104, no. 12, pp. 1655-1661, 1999.

[36] R. Alexanian, B. Barlogie, and S. Tucker, "VAD-based regimens as primary treatment for multiple myeloma," The American Journal of Hematology, vol. 33, no. 2, pp. 86-89, 1990.

[37] K. Koskela, T. T. Pelliniemi, and K. Remes, "VAD regimen in the treatment of resistant multiple myeloma: slow or fast infusion?" Leukemia and Lymphoma, vol. 10, no. 4-5, pp. 347-351, 1993.
[38] W. Stenzinger, A. Blomker, W. Hiddemann, and J. van de Loo, "Treatment of refractory multiple myeloma with the vincristine-adriamycin-dexamethasone (VAD) regimen," Blut, vol. 61, no. 2-3, pp. 55-59, 1990.

[39] A. Bhardwaj, G. Sethi, S. Vadhan-Raj et al., "Resveratrol inhibits proliferation, induces apoptosis, and overcomes chemoresistance through down-regulation of STAT3 and nuclear factor$\kappa \mathrm{B}$-regulated antiapoptotic and cell survival gene products in human multiple myeloma cells," Blood, vol. 109, no. 6, pp. 22932302, 2007.

[40] M. Bhutani, A. K. Pathak, A. S. Nair et al., "Capsaicin is a novel blocker of constitutive and interleukin-6-inducible STAT3 activation," Clinical Cancer Research, vol. 13, no. 10, pp. 30243032, 2007.

[41] H. R. Stennicke and G. S. Salvesen, "Properties of the caspases," Biochimica et Biophysica Acta, vol. 1387, no. 1-2, pp. 17-31, 1998.

[42] M. O. Hengartner, "The biochemistry of apoptosis," Nature, vol. 407, no. 6805, pp. 770-776, 2000.

[43] K. Shuai, G. R. Stark, I. M. Kerr, and J. E. Darnell Jr., "A single phosphotyrosine residue of Stat91 required for gene activation by interferon- $\gamma$," Science, vol. 261, no. 5129, pp. 1744-1746, 1993.

[44] B. B. Aggarwal, G. Sethi, S. A. Kwang et al., "Targeting signaltransducer-and-activator-of-transcription-3 for prevention and therapy of cancer: modern target but ancient solution," Annals of the New York Academy of Sciences, vol. 1091, pp. 151-169, 2006.

[45] S. A. Kwang, G. Sethi, B. Sung, A. Goel, R. Ralhan, and B. B. Aggarwal, "Guggulsterone, a farnesoid X receptor antagonist, inhibits constitutive and inducible STAT3 activation through induction of a protein tyrosine phosphatase SHP-1," Cancer Research, vol. 68, no. 11, pp. 4406-4415, 2008.

[46] M. K. Pandey, S. Bokyung, S. A. Kwang, and B. B. Aggarwal, "Butein suppresses constitutive and inducible signal transducer and activator of transcription (stat) 3 activation and stat3regulated gene products through the induction of a protein tyrosine phosphatase SHP-1," Molecular Pharmacology, vol. 75, no. 3, pp. 525-533, 2009.

[47] B. B. Aggarwal, S. Shishodia, S. K. Sandur, M. K. Pandey, and G. Sethi, "Inflammation and cancer: how hot is the link?" Biochemical Pharmacology, vol. 72, no. 11, pp. 1605-1621, 2006.

[48] D. Xu and C. K. Qu, "Protein tyrosine phosphatases in the JAK/STAT pathway," Frontiers in Bioscience, vol. 13, no. 13, pp. 4925-4932, 2008.

[49] R. J. Chan, S. A. Johnson, Y. Li, M. C. Yoder, and G. S. Feng, "A definitive role of Shp-2 tyrosine phosphatase in mediating embryonic stem cell differentiation and hematopoiesis," Blood, vol. 102, no. 6, pp. 2074-2080, 2003.

[50] R. J. Shaw and L. C. Cantley, "Ras, PI(3)K and mTOR signalling controls tumour cell growth," Nature, vol. 441, no. 7092, pp. 424-430, 2006.

[51] X. Cao, F. Kambe, M. Yamauchi, and H. Seo, “Thyroidhormone-dependent activation of the phosphoinositide 3kinase/Akt cascade requires Src and enhances neuronal survival," Biochemical Journal, vol. 424, no. 2, pp. 201-209, 2009.

[52] N. Gao, Z. Zhang, B. H. Jiang, and X. Shi, "Role of PI3K/ $\mathrm{AKT} / \mathrm{mTOR}$ signaling in the cell cycle progression of human prostate cancer," Biochemical and Biophysical Research Communications, vol. 310, no. 4, pp. 1124-1132, 2003.

[53] X. Wang, P. Yue, A. K. Young, H. Fu, F. R. Khuri, and S. Y. Sun, "Enhancing mammalian target of rapamycin (mTOR)targeted cancer therapy by preventing mTOR/raptor inhibitioninitiated, mTOR/rictor-independent Akt activation," Cancer Research, vol. 68, no. 18, pp. 7409-7418, 2008. 
[54] J. C. Soria, F. A. Shepherd, J. Y. Douillard et al., "Efficacy of everolimus (RAD001) in patients with advanced NSCLC previously treated with chemotherapy alone or with chemotherapy and EGFR inhibitors," Annals of Oncology, vol. 20, no. 10, pp. 1674-1681, 2009.

[55] K. J. Pandya, S. Dahlberg, M. Hidalgo et al., "A randomized, phase II trial of two dose levels of temsirolimus (CCI-779) in patients with extensive-stage small-cell lung cancer who have responding or stable disease after induction chemotherapy: a trial of the Eastern cooperative oncology group (E1500)," Journal of Thoracic Oncology, vol. 2, no. 11, pp. 1036-1041, 2007.

[56] M. Hidalgo, J. C. Buckner, C. Erlichman et al., "A phase I and pharmacokinetic study of temsirolimus (CCI-779) administered intravenously daily for 5 days every 2 weeks to patients with advanced cancer," Clinical Cancer Research, vol. 12, no. 19, pp. 5755-5763, 2006.

[57] A. Carracedo, L. Ma, J. Teruya-Feldstein et al., "Inhibition of mTORC1 leads to MAPK pathway activation through a PI3Kdependent feedback loop in human cancer," Journal of Clinical Investigation, vol. 118, no. 9, pp. 3065-3074, 2008.

[58] F. Zunino, R. Gambetta, and A. Di Marco, "The inhibition in vitro of DNA polymerase and RNA polymerases by daunomycin and adriamycin," Biochemical Pharmacology, vol. 24, no. 2, pp. 309-311, 1975.

[59] K. M. Tewey, T. C. Rowe, L. Yang, B. D. Halligan, and L. F. Liu, "Adriamycin-induced DNA damage mediated by mammalian DNA topoisomerase II," Science, vol. 226, no. 4673, pp. 466-468, 1984.

[60] S. W. Wong, A. F. Wahl, P. M. Yuan et al., "Human DNA polymerase alpha gene expression is cell proliferation dependent and its primary structure is similar to both prokaryotic and eukaryotic replicative DNA polymerases," EMBO Journal, vol. 7, no. 1, pp. 37-47, 1988.

[61] K. Sugimoto, K. Yamada, M. Egashira et al., "Temporal and spatial distribution of DNA topoisomerase II alters during proliferation, differentiation, and apoptosis in HL-60 cells," Blood, vol. 91, no. 4, pp. 1407-1417, 1998.

[62] E. Czeczuga-Semeniuk, S. Wołczyński, M. Dabrowska, J. Dzieciol, and T. Anchim, "The effect of doxorubicin and retinoids on proliferation, necrosis and apoptosis in MCF-7 breast cancer cells," Folia Histochemica et Cytobiologica, vol. 42, no. 4, pp. 221-227, 2004.

[63] T. Efferth, M. Giaisi, A. Merling, P. H. Krammer, and M. Li-Weber, "Artesunate induces ROS-mediated apoptosis in doxorubicin-resistant T leukemia cells," PloS ONE, vol. 2, no. 1, article e693, 2007. 


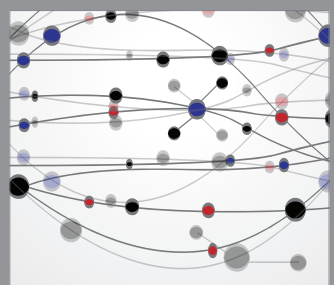

The Scientific World Journal
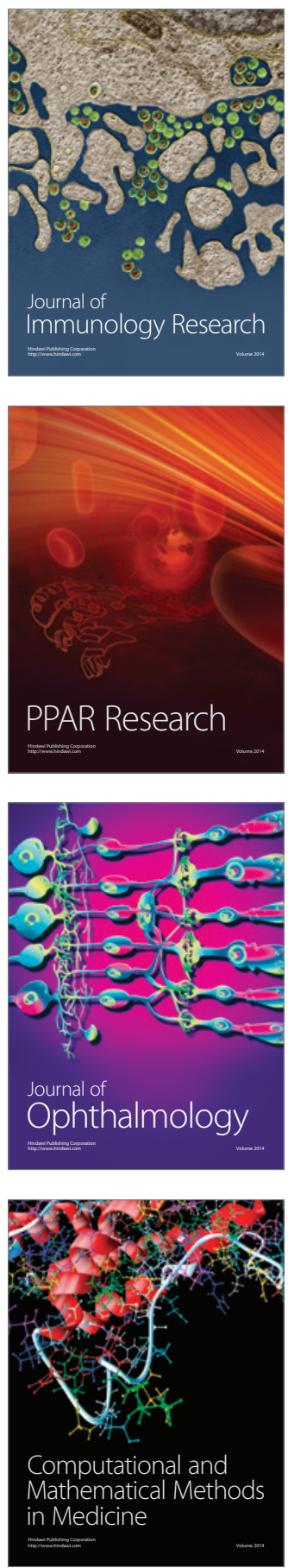

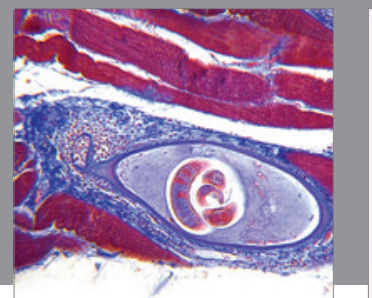

Gastroenterology

Research and Practice
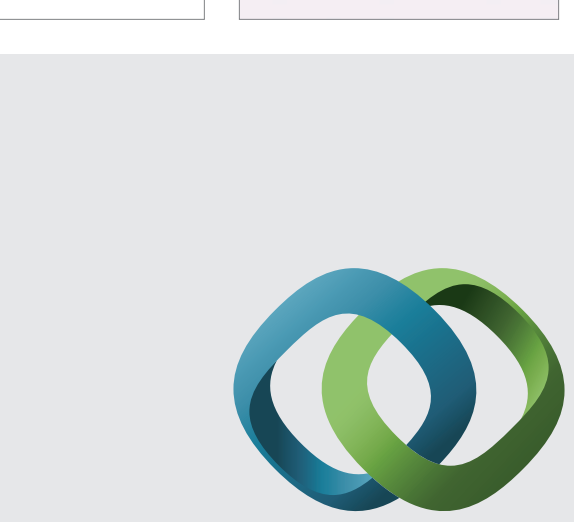

\section{Hindawi}

Submit your manuscripts at

http://www.hindawi.com
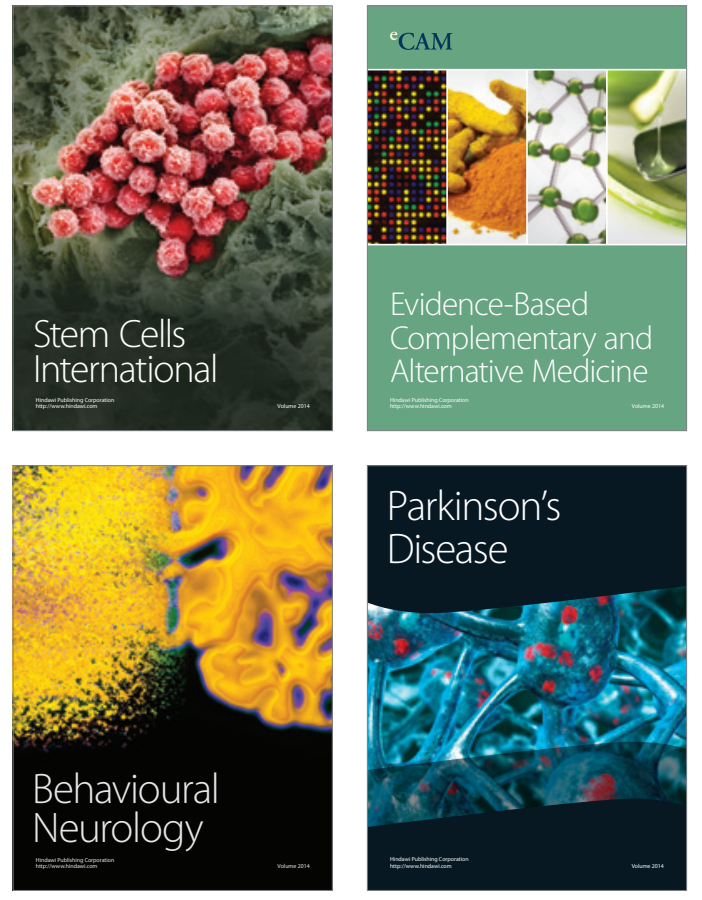
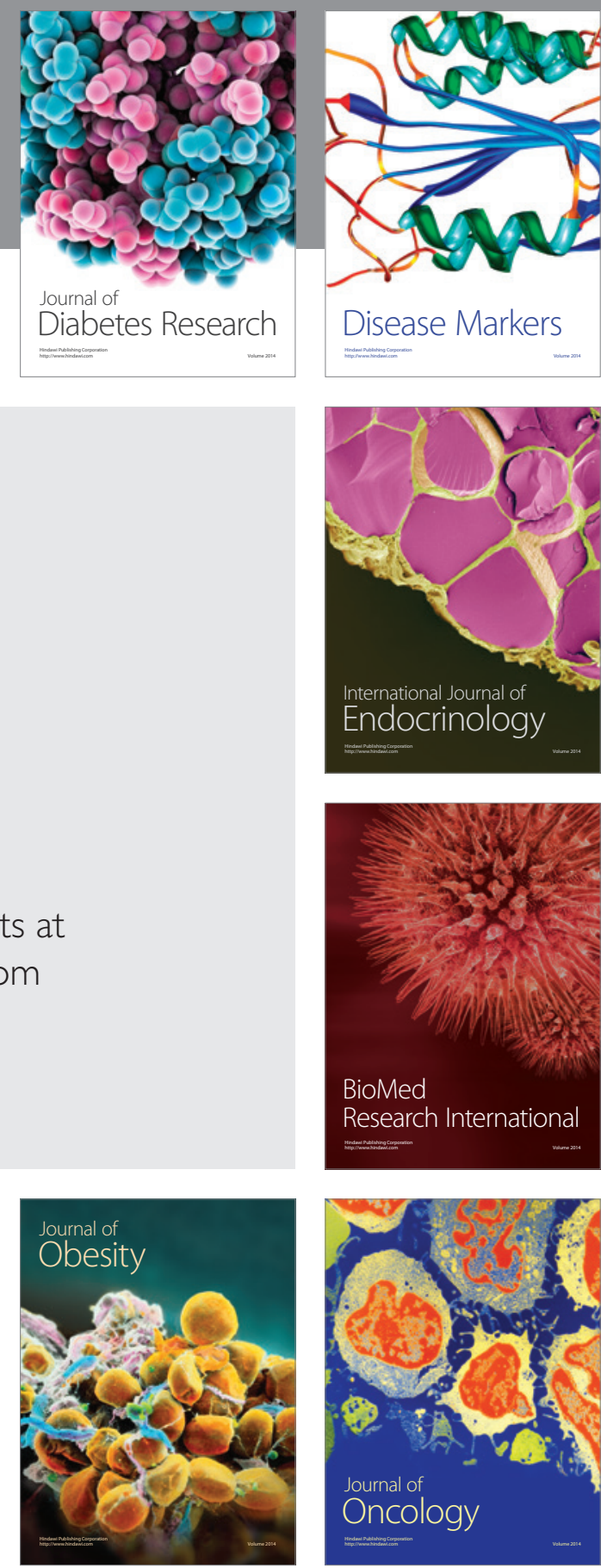

Disease Markers
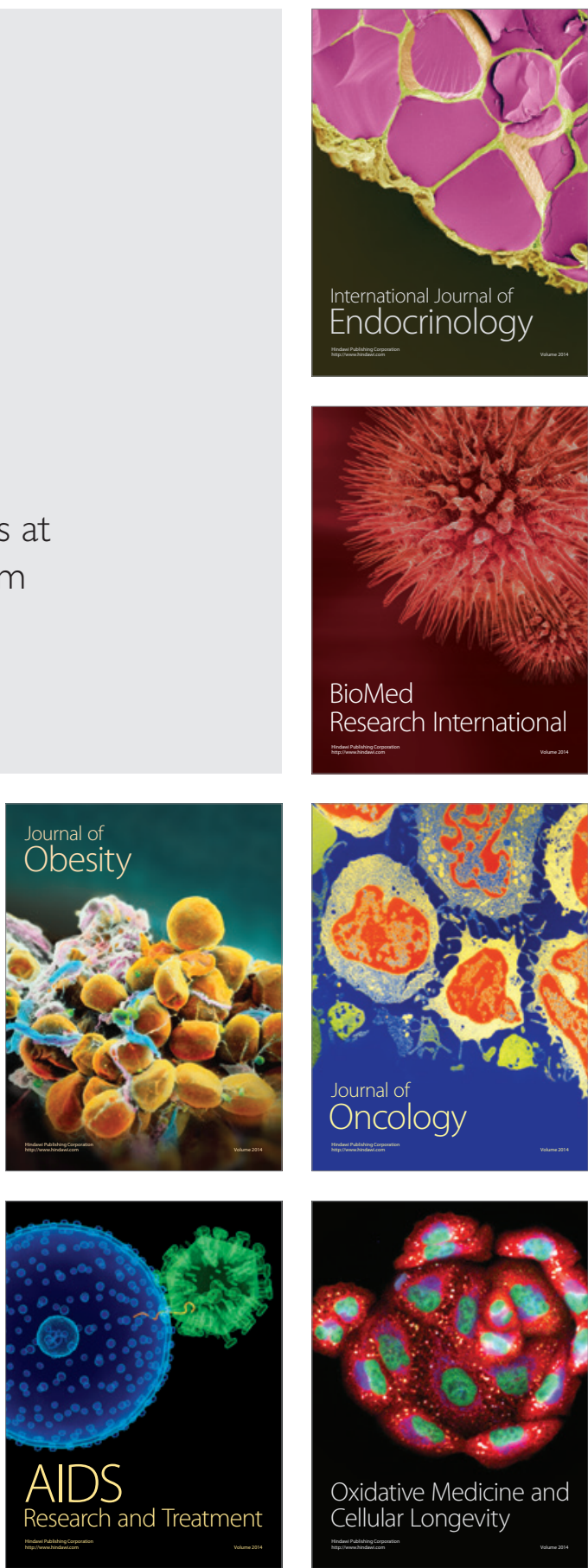